\title{
Molecular Dynamics Simulation on the Conformational Transition of the Mad2 Protein from the Open to the Closed State
}

\section{Chaoqun $\mathrm{Li}^{1}$, Yanyan Zhu ${ }^{2}$, Yan Wang ${ }^{1, *}$ and Guangju Chen ${ }^{1, *}$}

1 College of Chemistry, Beijing Normal University, 19 Xinjiekouwai Street, Beijing 100875, China; E-Mail: lichaoqun_210@163.com

2 Department of Chemistry, Zhengzhou University, Zhengzhou 450052, Henan, China; E-Mail: zhuyan@zzu.edu.cn

* Authors to whom correspondence should be addressed; E-Mails: wangy@bnu.edu.cn (Y.W.); gjchen@bnu.edu.cn (G.C.); Tel.: +86-10-5880-5247 (Y.W.); Fax: +86-10-5880-2075 (Y.W.).

Received: 19 December 2013; in revised form: 21 February 2014 / Accepted: 21 March 2014 / Published: 31 March 2014

\begin{abstract}
The Mad2 protein, with two distinct conformations of open- and closed-states, is a key player in the spindle checkpoint. The closed Mad2 state is more active than the open one. We carried out conventional and targeted molecular dynamics simulations for the two stable Mad2 states and their conformational transition to address the dynamical transition mechanism from the open to the closed state. The intermediate structure in the transition process shows exposure of the $\beta 6$ strand and an increase of space around the binding sites of $\beta 6$ strand due to the unfolding of the $\beta 7 / 8$ sheet and movement of the $\beta 6 / 4 / 5$ sheet close to the $\alpha \mathrm{C}$ helix. Therefore, Mad2 binding to the $\mathrm{Cdc} 20$ protein in the spindle checkpoint is made possible. The interconversion between these two states might facilitate the functional activity of the Mad2 protein. Motion correlation analysis revealed the allosteric network between the $\beta 1$ strand and $\beta 7 / 8$ sheet via communication of the $\beta 5-\alpha \mathrm{C}$ loop and the $\beta 6 / 4 / 5$ sheet in this transition process.
\end{abstract}

Keywords: molecular dynamics simulations; open/closed Mad2; dynamical transition mechanism 


\section{Introduction}

The spindle assembly checkpoint (SAC) proteins monitor a series of events in mitosis to ensure the accuracy of chromosome segregation prior to cell division, and are indispensable for chromosome stability [1-4]. Mitotic arrest deficiency $2(\operatorname{Mad} 2)$, which is a critical molecular component of the SAC proteins, is able to inhibit the anaphase-promoting complex or cyclosome (APC/C), combining with $\mathrm{Cdc} 20$ (an activator of $\mathrm{APC} / \mathrm{C}$ ) to delay separation of premature sister-chromatid through the binding of Mad2 to Cdc20 [5-14]. Premature sister-chromatid separation leads to aneuploidy (abnormal number of chromosomes), which contributes to cancer progression [5,15-18]. Mad2 binding to Cdc20 is helpful for accurate chromosome segregation to prevent cancer development. Therefore, it is important that the active Mad2 protein binds to $\mathrm{Cdc} 20$ in the mitotic process.

Usually, a given protein has a unique and native three-dimensional structure with the lowest possible Gibbs free energy [19]. However, Mad2 shows the characteristics of a "metamorphic" protein, and is able to adopt two distinctly different conformations, i.e., the open and closed Mad2 states under native conditions [20-22]. The first high-resolution X-ray crystal structure of the open Mad2 state was determined by Luo et al. in 2000 [23]. Since then, significant progress has been made in filling the structural space of this family. Luo et al. again reported the structures of several closed Mad2 states in 2002 and 2004 [20,24]. Subsequently, other crystal structures of the Mad2 monomer and dimer were also reported $[14,25,26]$. These crystal structures revealed that the structure of the closed Mad2 state is strikingly different from the open Mad2 state. In the open Mad2 state, the structure of Mad2 consists of three layers: a central layer formed by three $\alpha$-helices $(\alpha \mathrm{A}, \alpha \mathrm{B}$ and $\alpha \mathrm{C})$, a large six $\beta$-sheet ordered as $\beta 7-\beta 8-\beta 6-\beta 4-\beta 5-\beta 1$ in the front layer, and a short $\beta$-hairpin $(\beta 2, \beta 3)$ in the back layer. In the closed $\operatorname{Mad} 2$ state, the predominant differences in features of this state with respect to the open one are the large five $\beta$-sheet ordered as $\beta 6-\beta 4-\beta 5-\beta 8 "-\beta 8$ ' and a $N$-terminal $\beta 1$ strand forming an additional helix attached at the $\alpha \mathrm{A}$ helix. Moreover, the $\beta 6$ strand is exposed in the closed Mad2 state, while it is blocked in the open Mad2 state. It has been reported that $\mathrm{Cdc} 20$ easily forms an edge-on interaction with the $\beta 6$ strand at the ligand-binding site in the closed Mad2 state. The closed Mad2 monomer or dimer is more functionally active than the open one, which might facilitate Cdc20 binding in order to impart fidelity chromosome segregation.

Cytosolic Mad2 in human cells is mainly represented by an open Mad2 monomer [20]. Then, the active closed Mad 2 conformation should be initially transformed by the open Mad 2 monomer with or without the Mad1-Mad2 template [27-31]. Therefore, the conversion of the open state to the closed state in the Mad2 protein plays a key role for Cdc20 binding and has become an area of considerable interest over the past decades. It is well-known that the open Mad2 state is able to spontaneously convert to the closed Mad2 state [20]. Based on the difference between the open and closed states, their conversion should involve the transformation of the $\beta 7 / 8$ sheet and $\beta 1$ strand. Some experimental data suggests that the open Mad2 state undergoes a conformational change to the closed state via a high-energy intermediate conformation, which might favor Cdc20 binding [26,32]. On the other hand, the theoretical investigations on the Mad2 protein are very limited so far. Therefore, it remains unclear what the dynamical transition mechanism of the two states is and what the exact allosteric network in this conversion is. Because it is difficult to experimentally isolate the intermediate structures during the 
conformational transition process, it is highly valuable to explore the conformational transition process in more detail by performing computational simulations at the atomic level.

In the present study, we carried out conventional molecular dynamics (CMD) and targeted molecular dynamics (TMD) simulations in order to explore the conformational transition pathway in more detail. The results obtained from the simulations provide valuable insights into this conformational transition process with respect to how one state gradually transforms into another.

\section{Results}

The initial structures of the open and closed Mad2 protein states (denoted as O-Mad2 and C-Mad2, respectively, for convenience) used in the CMD simulations were based on previously reported and deposited X-ray crystal structures (PDB codes 1DUJ and 2VFX (K chain)) [14,23]. The O-Mad2 model consists of three $\alpha$ helices $(\alpha \mathrm{A}: 18-37, \alpha \mathrm{B}: 61-75$, and $\alpha \mathrm{C}: 122-142)$ and eight $\beta$ strands $(\beta 1$ : $11-13, \beta 2$ : 43-49, $\beta 3$ : 53-58, $\beta 4: 81-90, \beta 5: 97-105, \beta 6: 150-160, \beta 7: 170-174$ and $\beta 8$ : $184-191)$. The missing residues (Met1-Gly10, Met196-Asp205) in this model were built using the loop search method in the Swiss-Pdb Viewer (http://spdbv.vital-it.ch/). The C-Mad2 model consists of three $\alpha$ helices $(\alpha \mathrm{A}: 12-37, \alpha \mathrm{B}: 61-75$, and $\alpha \mathrm{C}: 122-142)$ and seven $\beta$ strands $(\beta 2$ : $43-49, \beta 3: 53-58$, $\beta 4: 81-90, \beta 5: 97-105, \beta 6: 150-160, \beta 8$ ': $177-188$, and $\beta 8 ": 190-200)$. The mutated residue Ala13 in the $\mathrm{C}-\mathrm{Mad} 2$ model was modeled by starting from the original residue Leu13.

To explore the detailed conformational transition pathway, we first carried out CMD simulations on the O-Mad2 and C-Mad2 states to understand the stability of the two Mad2 structures, followed by TMD simulations associated with the transition from the open state to the closed state and vice versa to explore the dynamic transition between the two states of the protein. Depicted in Figure 1 are the plots for the time-dependent root mean square deviations (RMSDs) for the positions of all backbone atoms in the CMD-simulated O-Mad2 and C-Mad2 structures, revealing that the CMD trajectories tended to be stabilized after about $5 \mathrm{~ns}$; the corresponding energies also tended to be stabilized after about 5 ns. Therefore, for each protein state, trajectory analysis was performed to extract the equilibrated conformation between 30 and $50 \mathrm{~ns}$ simulation time, recording 10,000 snapshots (one for each $2 \mathrm{ps}$ ) for each trajectory. Depicted in Figures 2 and S1 are the plots for the RMSDs of the positions of all backbone atoms in the TMD-simulated protein structures from the corresponding final structures associated with the two TMD trajectories for the $\mathrm{O}-\mathrm{Mad} 2 \leftrightarrow \mathrm{C}-\mathrm{Mad} 2$ conversion. The plots depicted in Figures 2 and S1 reveal that the backbone atoms in the two TMD simulations all reached the target conformations within $12 \mathrm{~ns}$. The detailed structural transition results obtained from the CMD and TMD simulations are discussed below in detail. 
Figure 1. Root mean square deviation (RMSD) values of all backbone atoms of the $\mathrm{O}-\mathrm{Mad} 2$ and C-Mad2 models with respect to the corresponding starting structure for the simulations of O-Mad2 and C-Mad2 models.

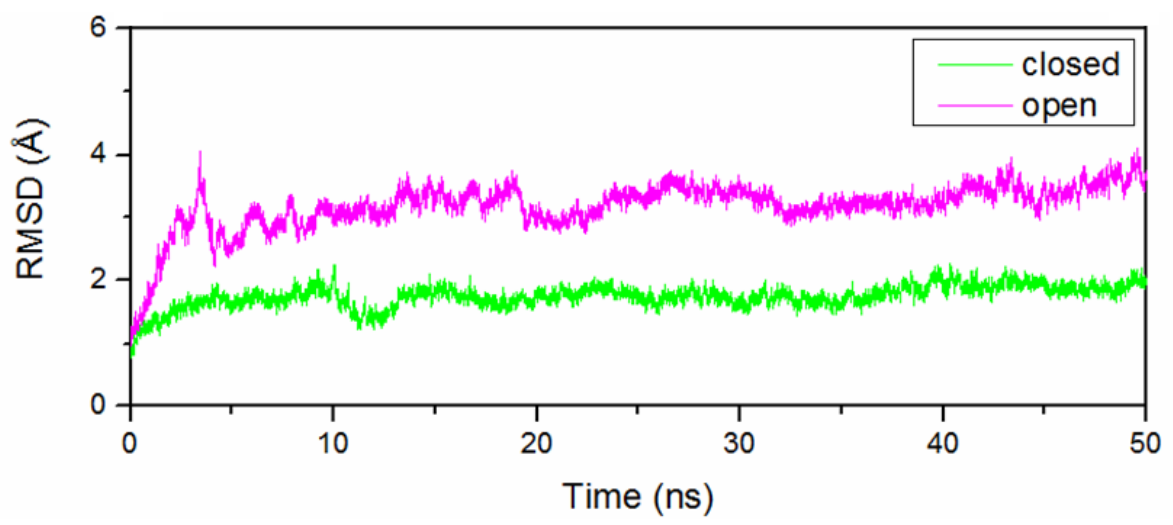

Figure 2. Root mean square deviation (RMSD) values of all backbone atoms and all atoms of the whole protein are shown for the O-Mad2 $\rightarrow$ C-Mad2 transition.

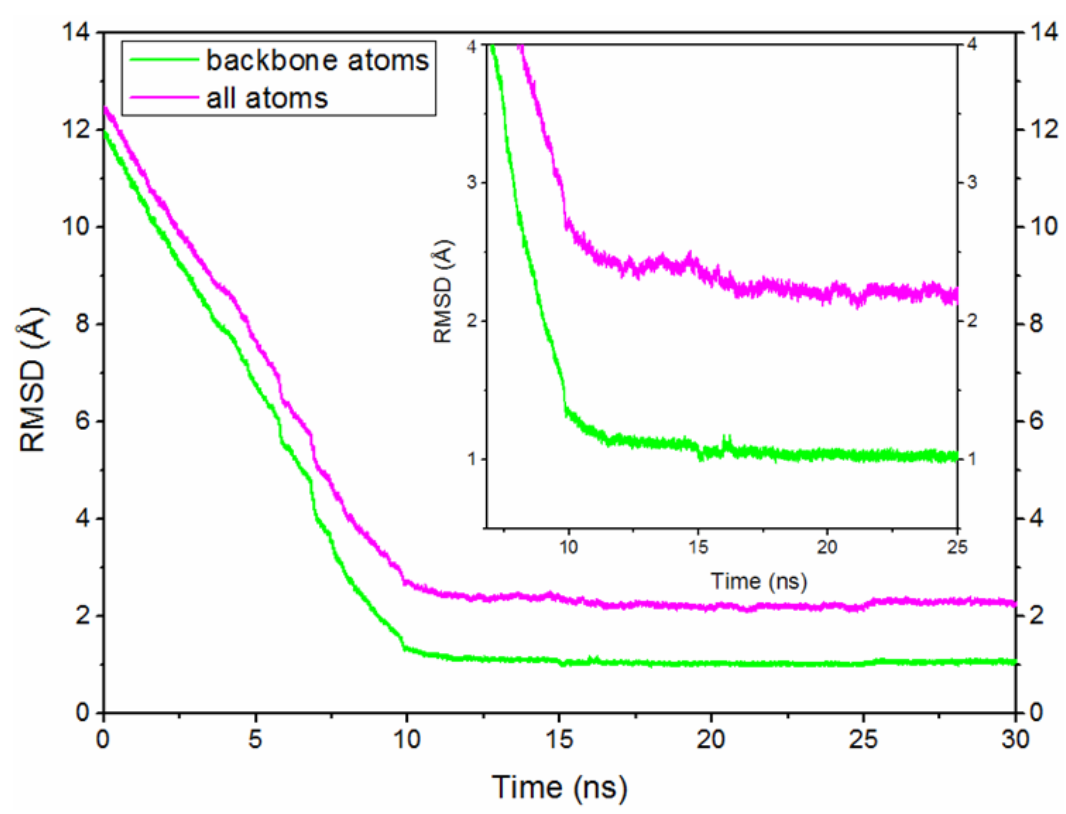

\subsection{Stability of the O-Mad2 and C-Mad2 States}

Based on the CMD simulations (see Figure 1), we obtained two dynamically stable states (the open and closed states) of Mad2. For each state, the average structure extracted from the CMD trajectory is shown in Figure 3. According to our analysis on the simulated structures, both the O-Mad2 and C-Mad2 structures simulated reflect the characteristics of the X-ray crystal structures very well. For example, these two states consist of three layers: a central layer formed by three $\alpha$-helices, a large six- or five-stranded $\beta$-sheet in the front layer, and a short $\beta$-hairpin in the back layer. Specifically, in the $\mathrm{O}-\mathrm{Mad} 2$ state, the three $\alpha$-helices in the central layer are $\alpha \mathrm{B}-\alpha \mathrm{A}-\alpha \mathrm{C}$, a large $\beta 7-\beta 8-\beta 6-\beta 4-\beta 5-\beta 1$ $\beta$-sheet in the front layer, and a short $\beta 2-\beta 3 \beta$-hairpin in the back layer arranged orderly from left to right in Figure 3a. In the C-Mad2 state, except for the large $\beta 6-\beta 4-\beta 5-\beta 8$ "- $-\beta 8^{\prime} \beta$-sheet rearranged in the front layer, similar components in the central and back layers can be found in Figure $3 \mathrm{~b}$. The most 
remarkable differences between the O-Mad2 and C-Mad2 states are: (1) the $\beta 7 / 8$ sheet is located on the left side of the $\beta$-sheet in $\mathrm{O}-\mathrm{Mad} 2$ and the $\beta 8^{\prime \prime} / 8^{\prime}$ sheet located to the right in $\mathrm{C}-\mathrm{Mad} 2$; (2) the $\beta 1$ strand in O-Mad2 forms a new helix attached to the $N$-terminus of $\alpha \mathrm{A}$ in $\mathrm{C}-\mathrm{Mad} 2$.

Figure 3. MD-simulated Mad2 structures for the open state (a) and closed state (b).

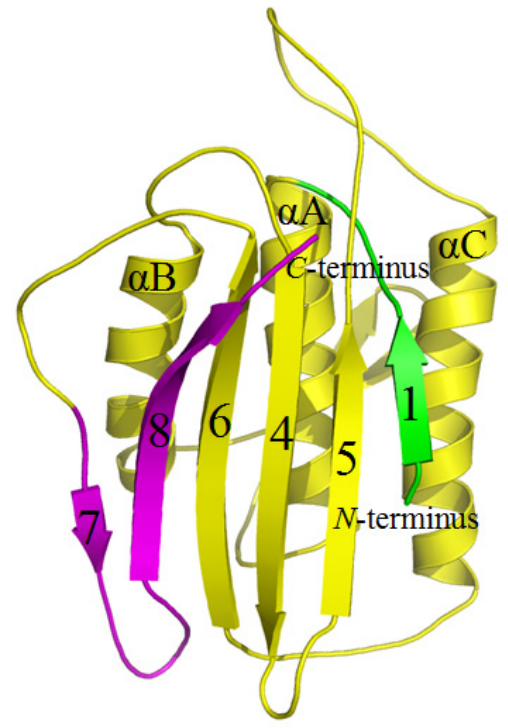

(a)

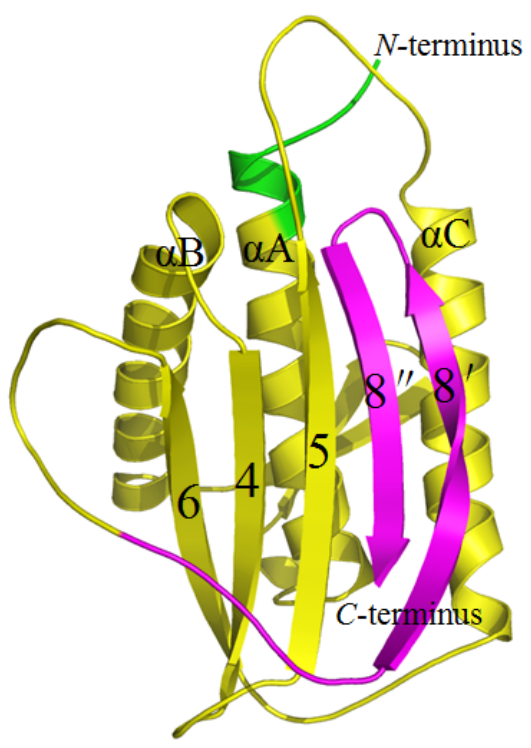

(b)

\subsection{The Conformational Transition from $\mathrm{O}-\mathrm{Mad} 2$ to $\mathrm{C}-\mathrm{Mad} 2$}

Based on the structural features of the metamorphic protein Mad2, we performed TMD simulations to explore the conformational transition process between O-Mad2 and C-Mad2. Based on the two TMD simulations for the O-Mad2 $\leftrightarrow \mathrm{C}-\mathrm{Mad} 2$ conversion, the forward and reverse transitions almost followed similar structural conversion processes computationally (see Figures 2, 4, S1 and S2). Consequently, we only present the conformational transition from O-Mad2 to C-Mad2 starting from the equilibrated $\mathrm{O}-\mathrm{Mad} 2$ structure. During the transition process, three transition conformations (I ( $\sim 3.5 \mathrm{~ns})$, II ( $\sim 6.5 \mathrm{~ns})$, III ( $\sim 9.5 \mathrm{~ns})$ ) of the Mad2 protein were extracted from the TMD simulation trajectory and were simulated via the CMD method as initial structures. The corresponding time-average structures are shown in Figure 4. In the first step of the transition (from O-Mad2 to I), the $\beta 1$ strand in the O-Mad2 state extends to a line loop and, simultaneously, the $\beta 7 / 8$ sheet unfolds to form conformation I with the destruction of their hydrogen bonds and hydrophobic interactions. In the second step of the transition (from I to II), the unfolded $\beta 1$ strand in conformation I traverses through the $\beta 5-\alpha \mathrm{C}$ loop close to the $\alpha \mathrm{A}$ helix and forms a new helix attached to the $N$-terminus of $\alpha \mathrm{A}$ helix to produce conformation II, which causes the large fluctuation of the $\beta 5-\alpha \mathrm{C}$ loop and favors the $\beta 7 / 8$ shift through emptying the right region of the $\beta 6 / 4 / 5$ sheet. Simultaneously, the $\beta 6 / 4 / 5$ sheet in the front layer moves close to the $\alpha \mathrm{C}$ helix in the central layer (see Figure 4), which results in the exposure of binding sites around the $\beta 6$ strand and an increase in the space between the $\beta 6$ strand and the unfolded $\beta 7 / 8$ sheet, ultimately increasing its activity to combine with the Cdc20 protein. Because the structural characteristics of II showed that the new helix, attached to the $N$-terminus of $\alpha \mathrm{A}$ helix is completely formed and that the $\beta 6 / 4 / 5$ sheet is closest to the $\alpha \mathrm{C}$ helix in the central layer 
(see Discussion Section), II was regarded as the intermediate during the conversion of O-Mad2 to C-Mad2. In the third step, i.e., the conformational transition from II to III, the unfolded $\beta 7 / 8$ sheet in conformation II moves from the left side of $\beta 6 / 4 / 5$ sheet to its front to produce conformation III. In the fourth step of the transition (from III to C-Mad2), the unfolded $\beta 7 / 8$ sheet in conformation III moves to the right side of the $\beta 6 / 4 / 5$ sheet to form a new $\beta 8^{\prime \prime} / 8^{\prime}$ sheet and produces the C-Mad2 state through formation of new hydrogen bonds and hydrophobic interactions (see Figure 4). In summary, the transition process from $\mathrm{O}-\mathrm{Mad} 2$ to $\mathrm{C}-\mathrm{Mad} 2$ involves the initial unfolding of the $\beta 7 / 8$ sheet followed by the transfer of the unfolded $\beta 7 / 8$ sheet. Moreover, the $\beta 1$ strand traversing through the $\beta 5-\alpha \mathrm{C}$ loop is a prerequisite for $\beta 7 / 8$ translocation. The intermediate II is characterized by the exposure of the $\beta 6$ strand and the increase in space of binding sites around $\beta 6$, which is possibly a favorable conformation to combine with the $\mathrm{Cdc} 20$ protein to mediate $\mathrm{APC} / \mathrm{C}$ inhibition.

Figure 4. The three-dimensional structures of the conformational transition pathway determined by TMD from O-Mad2 to C-Mad2.

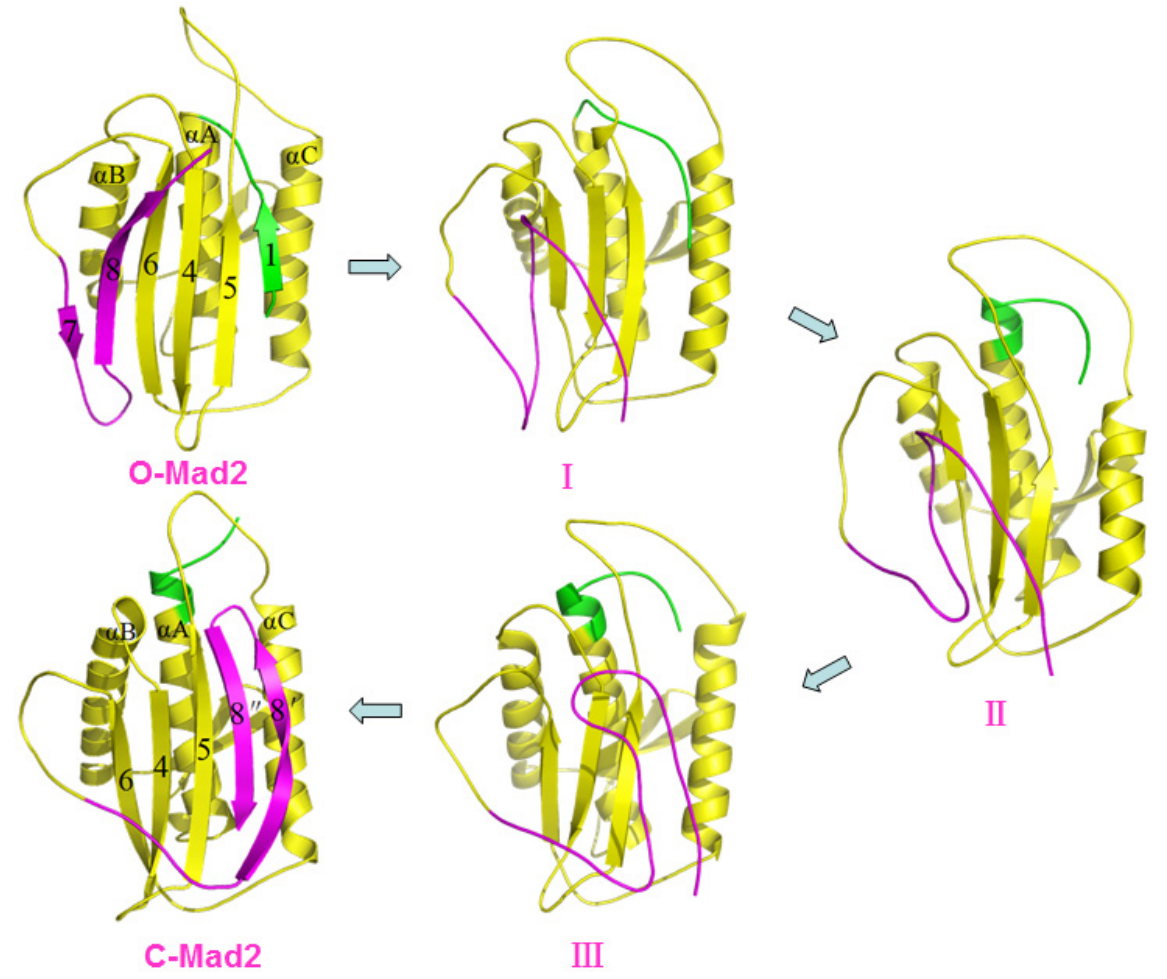

\subsection{Correlation Analysis along the Transition Pathway from O-Mad2 to C-Mad2}

To explore the allosteric communication of position changes for the $\beta 1$ strand and the $\beta 7 / 8$ sheet along the transition pathway from the O-Mad2 state to the C-Mad2 state, motion correlations of $\mathrm{C} \alpha$ atoms were analyzed and the corresponding cross-correlation map constructed from the trajectory is given in Figure 5 for the conversion of $\mathrm{O}-\mathrm{Mad} 2 \rightarrow \mathrm{C}-\mathrm{Mad} 2$. The results showed that the motion correlations between the residues ranged from high anticorrelation (blue) to high correlations (red). The results in Figure 5 demonstrate that the large correlated motions of the $\beta 1$ strand $v s$. the $\beta 5-\alpha \mathrm{C}$ loop occur remarkably with the large correlated motions of the $\beta 5-\alpha \mathrm{C}$ loop $v$ s. the $\beta 6 / 4 / 5$ sheet, which predicts that the allosteric communication between the $\beta 1$ strand and the $\beta 6 / 4 / 5$ sheet occurs via the 
$\beta 5-\alpha \mathrm{C}$ loop region. Furthermore, the motions of the $\beta 6 / 4 / 5$ sheet significantly correlate with the motions of the $\beta 7 / 8$ sheet, which predicts the allosteric communication between the $\beta 6 / 4 / 5$ and the $\beta 7 / 8$ sheets.

Figure 5. Dynamical cross-correlation map of the transition from O-Mad2 to C-Mad2 with specific sub-region squares in black.

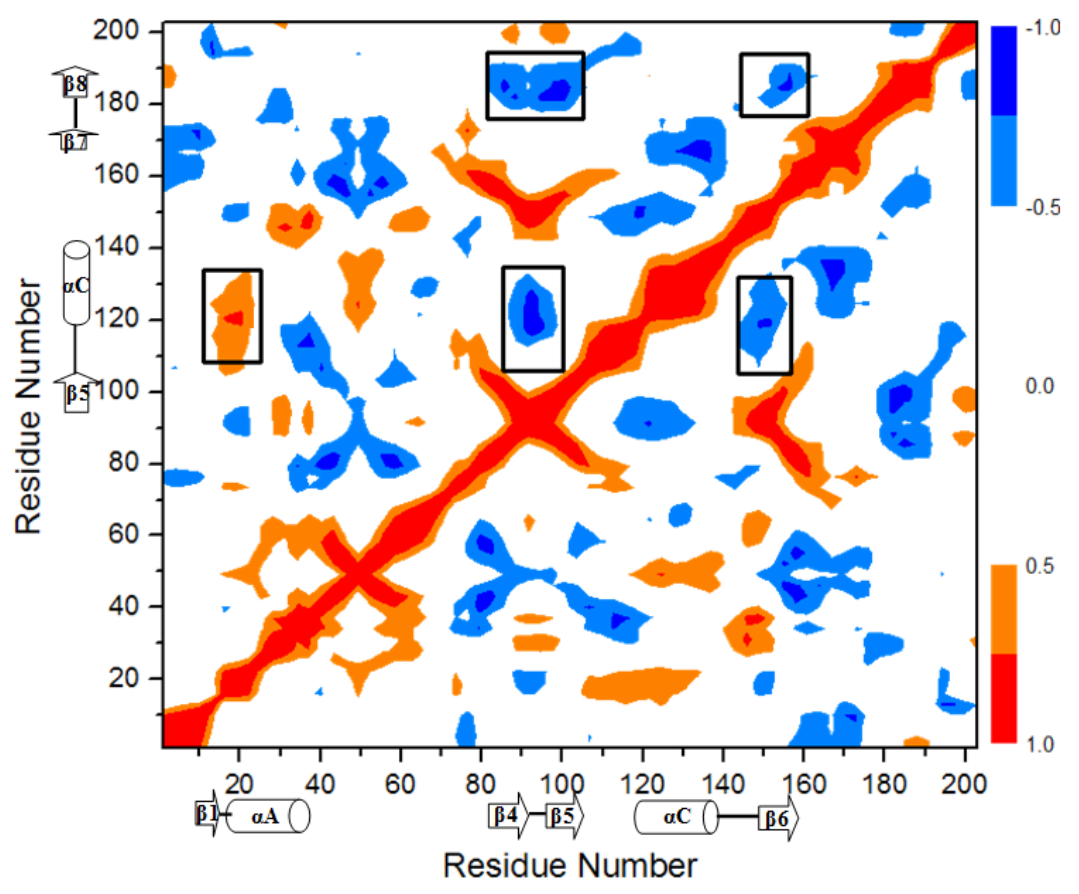

\section{Discussion}

\subsection{Conformational Characteristics along the Transition Pathway from O-Mad2 to C-Mad2}

To explore the details of the structural changes along the transition pathway from O-Mad2 to $\mathrm{C}$-Mad2, we used the $\alpha \mathrm{A}$ and $\alpha \mathrm{C}$ helices as reference points to calculate the variations in mass center distances between the $\beta 1$ strand and the $\alpha \mathrm{A}$ helix, between the $\beta 7 / 8$ sheet and the $\alpha \mathrm{C}$ helix, and between the $\beta 6 / 4 / 5$ sheet and the $\alpha \mathrm{C}$ helix. The corresponding results are shown in Figure 6 . It can be seen from the conversion of O-Mad2 to C-Mad2 that the distance between the $\beta 1$ strand and the $\alpha \mathrm{A}$ helix increases from 11.4 to $22.5 \AA$, which corresponds to the $\beta 1$ strand traversing through the $\beta 5-\alpha \mathrm{C}$ loop and the emptying the right region of the $\beta 6 / 4 / 5$ sheet, consequently favoring the $\beta 7 / 8$ translocation. Moreover, the distance between the $\beta 7 / 8$ sheet and the $\alpha \mathrm{C}$ helix decreases from $26.2 \AA$ in the O-Mad2 state to $14.8 \AA$ in the C-Mad2 state, which represents the movement of the $\beta 7 / 8$ sheet from the left side of the $\beta 6 / 4 / 5$ sheet to its right side. Interestingly, this distance variation is slower than that between the $\beta 1$ strand and the $\alpha \mathrm{A}$ helix (see Figure 6), which suggests that the $\beta 1$ strand traversing through the $\beta 5-\alpha \mathrm{C}$ loop occurs prior to the $\beta 7 / 8$ translocation, as discussed in Section 2.2. In particular, in the conformational transition from O-Mad2 to II, the distance between the $\beta 6 / 4 / 5$ sheet and the $\alpha \mathrm{C}$ helix decreases from $18.1 \AA$ in the $\mathrm{O}-\mathrm{Mad} 2$ state to $15.0 \AA$ in the intermediate II, which corresponds to the movement of the $\beta 6 / 4 / 5$ sheet in the front layer closest to the $\alpha \mathrm{C}$ helix in the central layer to form the intermediate. As expected, the moving distance of the $\beta 6 / 4 / 5$ sheet with $3.1 \AA$ 
predicts that the Cdc20 binding sites, i.e., residues Leu61, Tyr64, Asn67, Trp75, Asp152-Tyr156, Asp158, Asp160-Leu161, Val163, Lys166-Glu169, Pro172, Phe174 around the $\beta 6$ strand [24], in the intermediate could be considerably exposed compared with the O-Mad2 state (see Figure 7a). To further address the exposed space of Cdc20 binding sites in the intermediate, the CASTp program was used to calculate the size and shape of its pocket compared with that in the O-Mad2 state. Such analysis was shown to be able to provide a comprehensive and detailed quantitative characterization of interior voids and surface pockets of proteins [33-35]. Figure $7 \mathrm{~b}$ shows the binding site pockets in the O-Mad2 state and the intermediate, which are automatically identified by the CASTp analysis. The two topological equivalent cavities for the binding site pockets in the O-Mad2 state and the intermediate have different areas and volumes of $183.6 \AA^{2} / 146.0 \AA^{3}$ and $752.6 \AA^{2} / 735.3 \AA^{3}$, respectively. This result reveals that the size of the binding site pocket in the intermediate is much larger than in O-Mad2, which suggests that the intermediate is a favorable conformation for Cdc20 protein binding in the mitotic process. However, in the conformational transition from intermediate II to C-Mad2, the distance between the $\beta 6 / 4 / 5$ sheet and the $\alpha \mathrm{C}$ helix increases from 15.0 to $19.8 \AA$, which increases the locating space for the newly formed $\beta 8^{\prime \prime} / 8^{\prime}$ sheet and favors the $\beta 7 / 8$ translocation to form the $\beta 8^{\prime \prime} / 8^{\prime}$ sheet.

Figure 6. Mass center distances between the $\beta 6 / 4 / 5$ sheet and $\alpha \mathrm{C}$ helix (green), between the $\beta 7 / 8$ sheet and $\alpha \mathrm{C}$ helix (blue), and between the $\beta 1$ strand and $\alpha \mathrm{A}$ helix (red) along the transition pathway from O-Mad2 to C-Mad2. The roseate letters included in the grey areas denote the different conformations.

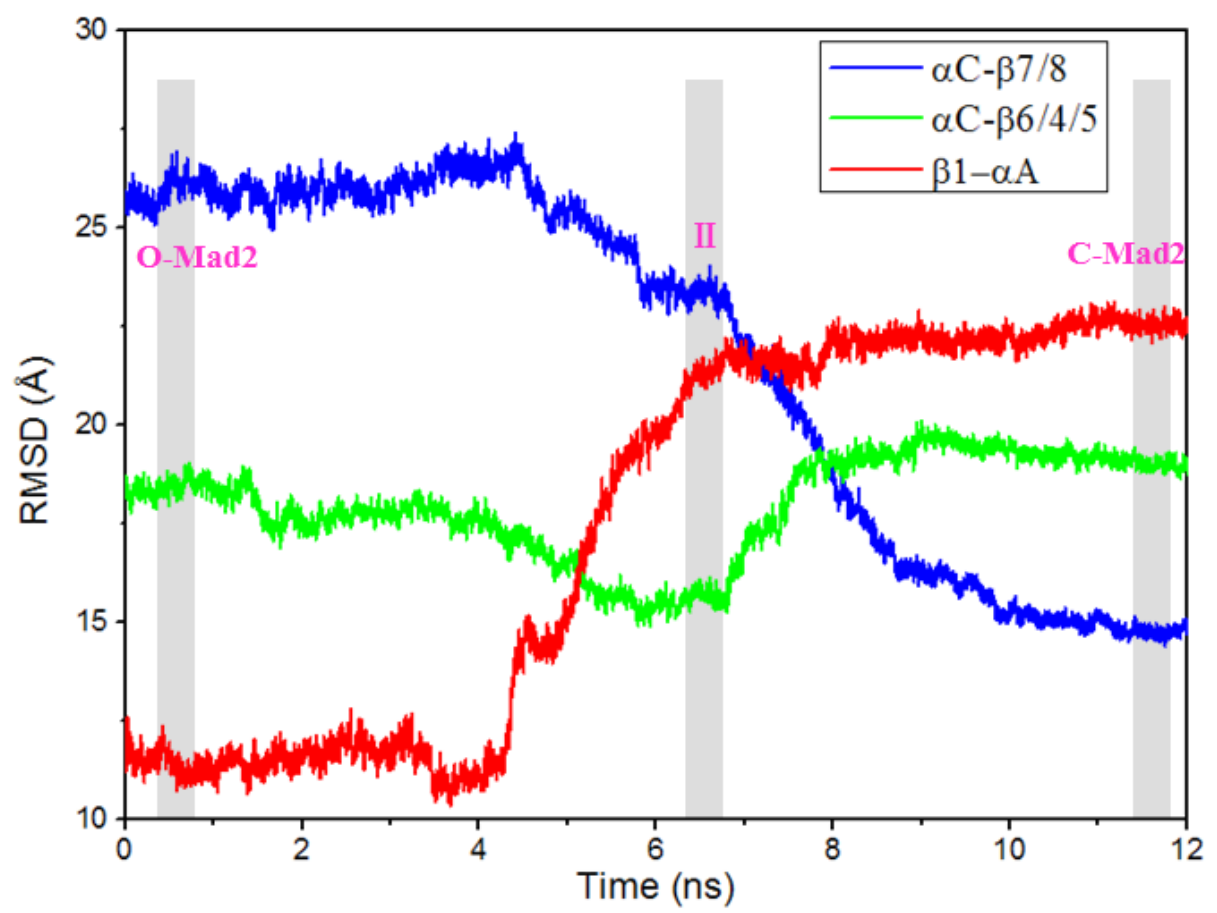


Figure 7. (a) Moving distance of the $\beta 6 / 4 / 5$ sheet between O-Mad2 and II; and (b) representation of the pockets detected by the CASTp program (cyan spheres: Cdc20 binding site pocket).

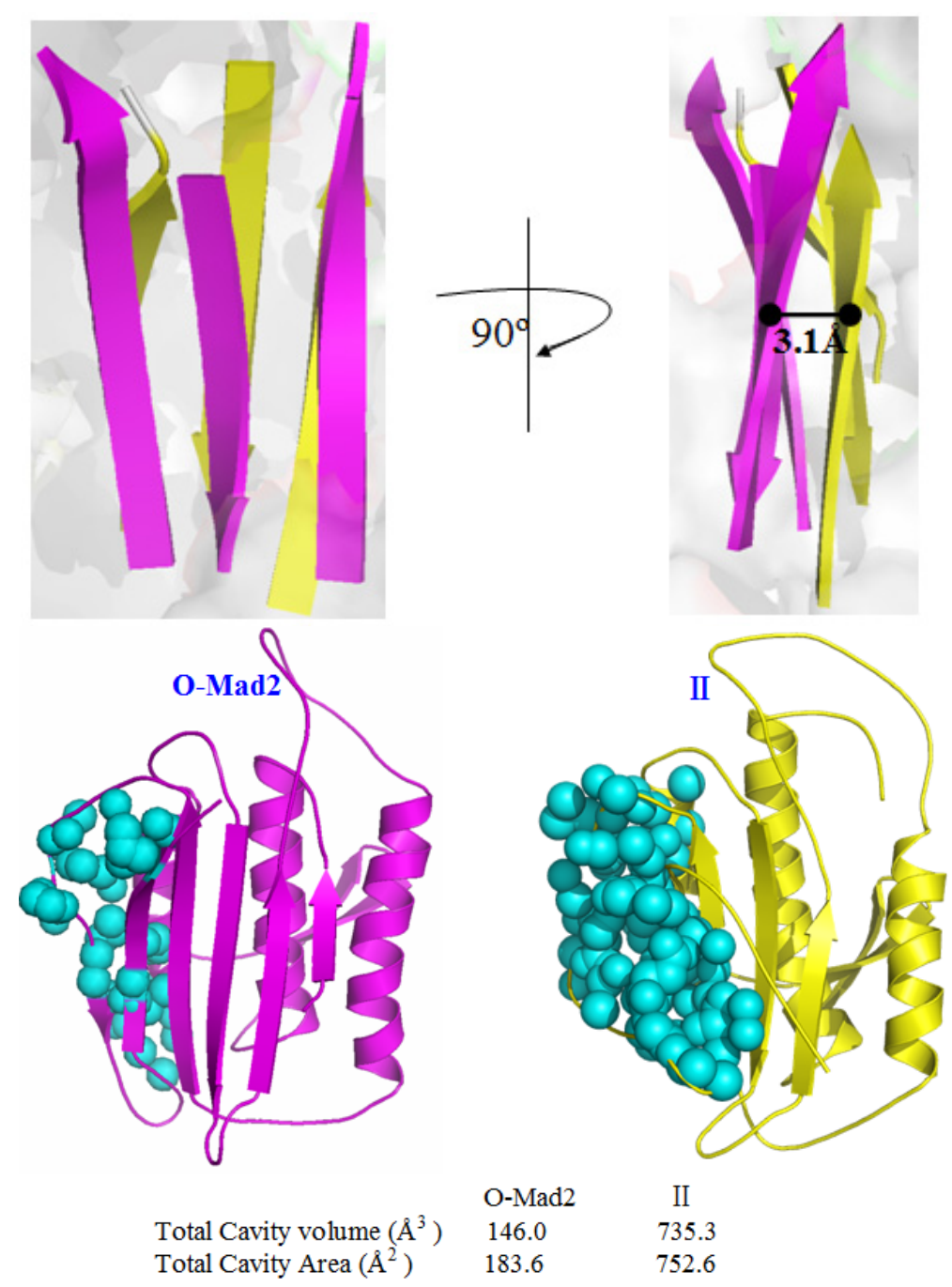

(a)

(b)

3.2. Hydrogen Bond and Hydrophobic Structure Analyses along the Transition Pathway from O-Mad2 to $C-M a d 2$

To gain information of hydrogen bonds and the hydrophobic core of Mad2, analyses of hydrogen bonds and hydrophobic contacts in the conversion process of O-Mad2 to C-Mad2 were performed. The occurrence of all possible hydrogen bonds for the $\beta 1$ strand and the $\beta 7 / 8$ sheet in the conversion process are shown in Table 1 by calculating the percentages of times on the corresponding CMD simulations. Based on the experimental observations, the hydrophobic core involves the residues Leu13 in $\beta 1$, Phe23 and Phe24 in $\alpha \mathrm{A}$, Phe102 in $\beta 5$, and Val193 in $\beta 8^{\prime \prime}$ [25]. Figure 8 shows the hydrophobic contacts at the hydrophobic core. A hydrophobic contact is defined as a distance between carbon atoms shorter than $4.5 \AA$. For the conformational transition from O-Mad2 to II, based on the unfolding of the $\beta 1$ strand and the $\beta 7 / 8$ sheet in the step of O-Mad2 to I (as discussed in Section 2.2), some hydrogen bonds that initially exist in the O-Mad2 state disappear gradually with the conformational change to I. For example, the hydrogen bond between the $\mathrm{N}-\mathrm{H}$ group of Asp101 in $\beta 5$ and the $\mathrm{O}$ atom of Gln9 in $\beta 1$ is maintained with the occupancy of $98 \%$ of simulation times in the 
O-Mad2 state, while the same hydrogen bond is maintained with the occupancy of $\sim 0 \%$ of simulation times in conformation I. Moreover, visual analysis shows that the hydrophobic contact distances of 3.2, 5.0 and $4.1 \AA$ between Leu13 and Phe23, between Leu13 and Phe24, and between Leu13 and Phe102 in the O-Mad2 state increase to $11.5,7.8$ and $12.9 \AA$, respectively, which predicts that hydrophobic interaction destruction occurs because Leu13 moves away during $\beta 1$ unfolding (see Figure 8). In the formation of the new helix at the $N$-terminus of the $\alpha \mathrm{A}$ helix in transition from I to II, as discussed, some hydrogen bonds are formed with the conformational change to II. For example, the hydrogen bond between the $\mathrm{N}-\mathrm{H}$ group of Ser16 and the $\mathrm{O}$ atom of Thr12 is maintained with the occupancy of $98.8 \%$ of simulation times in intermediate II, while this hydrogen bond is maintained with the occupancy of $\sim 0 \%$ of simulation times in conformation I.

Table 1. The occupancies (\%) of hydrogen bonds for the O-Mad2 state, I, II, III, and C-Mad2 state.

\begin{tabular}{|c|c|c|c|c|c|c|}
\hline Hydrogen bond & O-Mad2 & I & II & Hydrogen bond & III & C-Mad2 \\
\hline$(\mathrm{Ile} 11) \mathrm{N}-\mathrm{H} \cdots \mathrm{O}(\mathrm{G} \ln 101)$ & 98.2 & 0.0 & - & $\left(\right.$ Arg99) $\mathrm{N}-\mathrm{H}^{\cdots} \cdots \mathrm{O}(\mathrm{Ala} 198)$ & 0.0 & 99.6 \\
\hline$(\mathrm{Gln} 101) \mathrm{N}-\mathrm{H} \cdots \mathrm{O}(\mathrm{G} \ln 9)$ & 98.0 & 0.0 & - & $($ Arg99)O $\cdots \mathrm{H}-\mathrm{N}($ Ala198) & 0.0 & 95.6 \\
\hline$($ Asp 103)N-H $\cdots \mathrm{O}(\mathrm{Ile} 11)$ & 83.2 & 0.0 & - & $(\mathrm{Gln} 101) \mathrm{N}-\mathrm{H} \cdots \mathrm{O}(\mathrm{Met} 196)$ & 0.0 & 100.0 \\
\hline$(\mathrm{Phe151}) \mathrm{N}-\mathrm{H} \cdots \mathrm{O}(\operatorname{Ser} 185)$ & 98.0 & 0.0 & - & $(\mathrm{G} \ln 101) \mathrm{O} \cdots \mathrm{H}-\mathrm{N}($ Met196) & 0.0 & 98.8 \\
\hline$($ Leu 153)N-H $\cdots \mathrm{O}(\mathrm{Thr} 187)$ & 87.6 & 0.0 & - & $($ Asp103)N-H $\cdots \mathrm{O}($ Asn194) & 0.0 & 94.2 \\
\hline$($ Ile155)N-H $\cdots \mathrm{O}(\mathrm{Thr} 189)$ & 98.8 & 0.0 & - & $($ Asp103)O $\cdots \mathrm{H}-\mathrm{N}($ Asn194) & 0.0 & 99.6 \\
\hline$(\mathrm{Thr} 157) \mathrm{N}-\mathrm{H} \cdots \mathrm{O}(\mathrm{His} 191)$ & 89.8 & 0.0 & - & $($ Glu105)N-H $\cdots$ O(Lys 192) & 0.0 & 98.4 \\
\hline$(\mathrm{Thr} 188) \mathrm{N}-\mathrm{H} \cdots \mathrm{O}($ Pro172) & 56.6 & 0.0 & - & $($ Glu105)O $\cdots \mathrm{H}-\mathrm{N}($ Lys 192) & 0.0 & 100.0 \\
\hline$($ Thr189)N-H $\cdots \mathrm{O}($ Leu153) & 99.4 & 0.0 & - & (Glu179)N-H $\cdots$ O(Tyr199) & 0.0 & 99.4 \\
\hline$($ His191)N-H $\cdots \mathrm{O}(\mathrm{Ile} 155)$ & 99.4 & 0.0 & - & (Glu179)O $\cdots \mathrm{H}-\mathrm{N}(\mathrm{Tyr} 199)$ & 0.0 & 98.6 \\
\hline$(\mathrm{Thr} 187) \mathrm{N}-\mathrm{H} \cdots \mathrm{O}(\mathrm{Phe} 151)$ & 90.6 & 0.0 & - & $($ Val181)N-H $\cdots \mathrm{O}($ Val197) & 0.0 & 98.6 \\
\hline$(\mathrm{Phe1} 86) \mathrm{N}-\mathrm{H} \cdots \mathrm{O}(\mathrm{Phe} 174)$ & 88.0 & 0.0 & - & $($ Val181)O $\cdots \mathrm{H}-\mathrm{N}($ Val197) & 0.0 & 92.4 \\
\hline$(\mathrm{Phe1} 86) \mathrm{O} \cdots \mathrm{H}-\mathrm{N}(\mathrm{Phe} 174)$ & 97.8 & 0.0 & - & $($ Leu183)N-H $\cdots \mathrm{O}(\operatorname{Ser} 195)$ & 0.0 & 99.4 \\
\hline$($ Ser16)N-H $\cdots O(T h r 12)$ & - & 0.0 & 98.8 & $(\mathrm{Phe186)N}-\mathrm{H} \cdots \mathrm{O}($ Val193) & 0.0 & 99.4 \\
\hline$($ Ala17)N-H $\cdots \mathrm{O}($ Leu13) & - & 0.0 & 85.7 & $($ Phe186)O $\cdots \mathrm{H}-\mathrm{N}($ Val193) & 0.0 & 100.0 \\
\hline (Glu18)N-H $\cdots$ O(Arg14) & - & 0.0 & 57.9 & $(\mathrm{Thr} 188) \mathrm{N}-\mathrm{H} \cdots \mathrm{O}(\mathrm{His} 191)$ & 0.0 & 94.2 \\
\hline$($ Ile19)N-H $\cdots \mathrm{O}($ Gly15) & - & 0.0 & 88.4 & $($ Lys200)N-H $\cdots \mathrm{O}($ Leu97) & 0.0 & 94.2 \\
\hline$($ Val20)N-H $\cdots \mathrm{O}(\operatorname{Ser} 16)$ & - & 0.0 & 99.2 & & & \\
\hline$($ Ala21)N-H $\cdots \mathrm{O}($ Ala17) & - & 0.0 & 54.5 & & & \\
\hline
\end{tabular}

For the conformational transition from II to C-Mad2 in the formation of the new $\beta 8^{\prime \prime} / 8^{\prime}$ sheet in the transition of III to the C-Mad2 state, as discussed in Section 2.2, some new hydrogen bonds are formed with the conformational change to the C-Mad2 state. For example, the hydrogen bond of $\mathrm{NH}$ (Asp103) $-\mathrm{O}($ Asn194) in the $\beta 8 " / 8$ ' sheet with the occupancy of $94.2 \%$ of simulation times appears in the C-Mad2 state. Moreover, visual analysis shows that the hydrophobic contact distances of 13.7, 10.0, and $12.4 \AA$ between Val193 and Phe23, between Val193 and Phe24, and between Val193 and Phe102 decreases to 4.2, 4.3, and 4.1 $\AA$, respectively, in the C-Mad2 state, which predicts that a new hydrophobic interaction is formed with Val193 in the newly formed $\beta 8$ " moving into this hydrophobic core (see Figure 8). 
Figure 8. Representation of the hydrophobic interaction in the conformational transition pathway from O-Mad2 to C-Mad2.

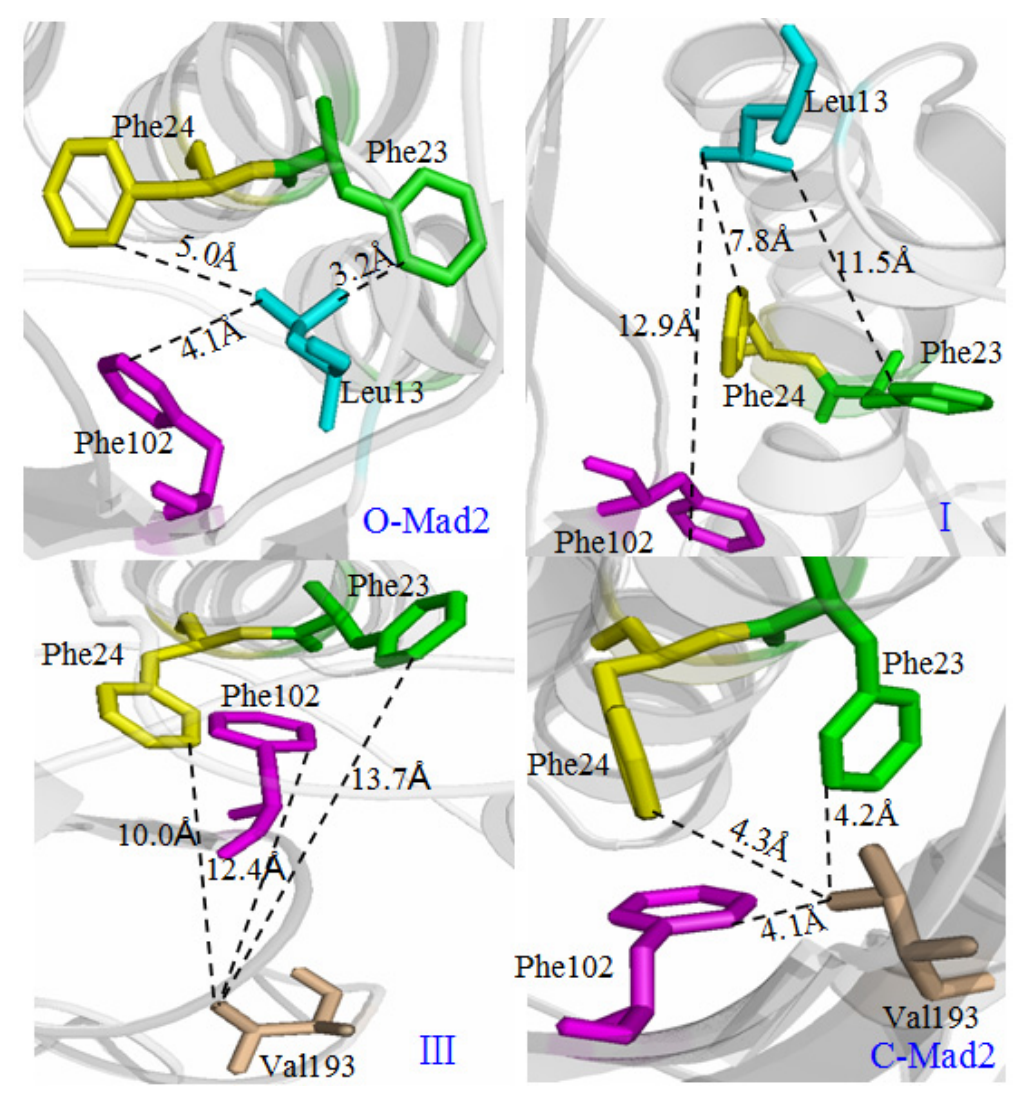

\subsection{Allosteric Network Analysis in the Conversion of O-Mad2 to C-Mad2}

To fully understand the allosteric communication in the conversion of the O-Mad2 state to the C-Mad2 state, we explored the relationship between the motion correlations and their conformational changes. It can be seen from the motion correlation analysis in Figure 5 that the $\beta 1$ strand correlates to the $\beta 5-\alpha C$ loop with large cross-correlate coefficients. Simultaneously, the $\beta 5-\alpha C$ loop correlates to the $\beta 6 / 4 / 5$ sheet; then the $\beta 6 / 4 / 5$ sheet further correlates to the $\beta 7 / 8$ sheet. Such communication transition represents the allosteric communication network from the $\beta 1$ strand to the $\beta 7 / 8$ sheet through the $\beta 5-\alpha \mathrm{C}$ loop and $\beta 6 / 4 / 5$ sheet. A coarse-grained picture of correlated segments of O-Mad2 and C-Mad2 is shown in Figure 9 with correlation connections. The width of the black-lines connecting correlated segments is proportional to the sum of the edges connecting them. The red-lines with arrowheads represent the correlation connections. It can be seen that the segment of the $\beta 1$ strand in the O-Mad2 state merges into the new segment of $\beta 1+\alpha \mathrm{A}$ in the C-Mad2 state, which induces the fluctuation of the $\beta 5-\alpha \mathrm{C}$ loop due to the fact that the $\beta 1$ strand traverses to the $N$-terminus of the $\alpha$ A helix through the $\beta 5-\alpha C$ loop. Such allosteric motion produces the correlation connection between the $\beta 1$ strand and the $\beta 5-\alpha \mathrm{C}$ loop. Because the $\beta 5$ strand and the $\alpha \mathrm{C}$ helix are connected to the $\beta 6$ strand and the $\beta 4$ strand, respectively, the movement of the $\beta 6 / 4 / 5$ sheet to the $\alpha \mathrm{C}$ helix relates definitely to the $\beta 5-\alpha \mathrm{C}$ loop, as expected for the correlations of the $\beta 5-\alpha \mathrm{C}$ loop to the $\beta 6 / 4 / 5$ sheet. Furthermore, the motion of the $\beta 6 / 4 / 5$ sheet facilitates the translocation of the $\beta 7 / 8$ sheet due to the connection of the $\beta 6 / 4 / 5$ sheet to the $\beta 7 / 8$ sheet, subsequently causing allosteric communication to the $\beta 7 / 8$ sheet. 
Figure 9. Color coded optimal correlated segment network of O-Mad2 (left) and C-Mad2 (right). Widths of the connecting black-lines are proportional to the intersegment edges. The red-lines with arrowheads and the red dot represent the correlation motion connections and the mass center of the $\beta 5-\alpha \mathrm{C}$ loop, respectively.

O-Mad2 C-Mad2

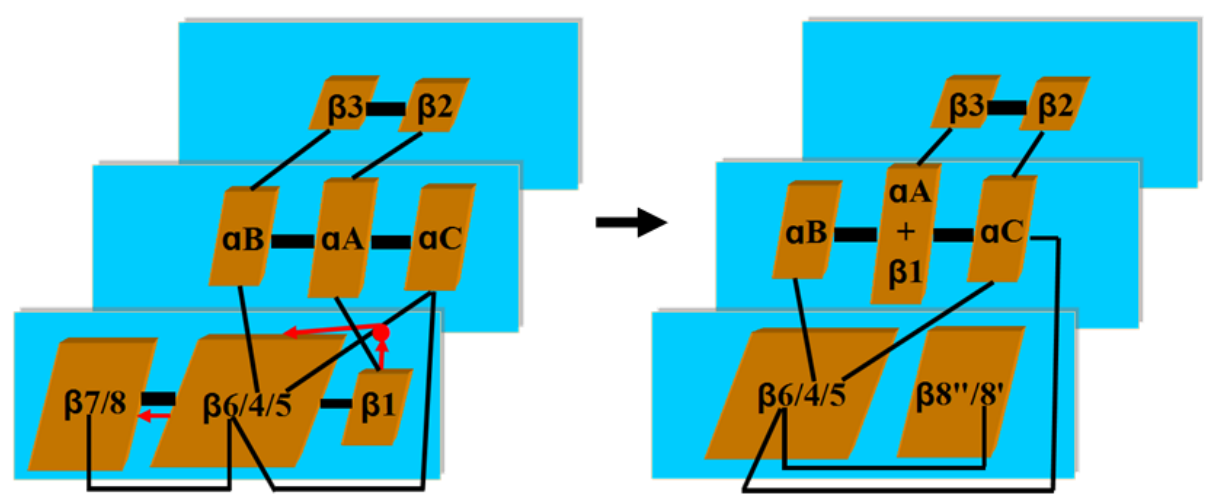

\section{Models and Methods}

\subsection{Conventional Molecular Dynamics Simulation}

All CMD simulations for these built models were carried out using the AMBER 9 package [36] and ff03 all atom force field parameters [37-39]. Five $\mathrm{Na}^{+}$ions were used to neutralize each of the O-Mad2 and C-Mad2 models, and an ionic strength of $50 \mathrm{mM}$ was generated by adding $9 \mathrm{Na}^{+}$and $9 \mathrm{Cl}^{-}$ions for these two models [14]. Similar counterion processes were applied to the other models. Each of the systems was explicitly solvated by using the TIP3P water potential inside an orthorhombic box of water molecules with a minimum solute-wall distance of $10 \AA$. The protocol for all CMD simulations is described as follows: (1) the systems were energetically minimized to remove unfavorable contacts. Four cycles of minimizations were performed with 5000 steps of each minimization and harmonic restraints on the Mad2 protein from $100,75,50$ to $25 \mathrm{kcal} /\left(\mathrm{mol} \cdot \AA^{2}\right)$, which means that the restraints were relaxed stepwise by $25 \mathrm{kcal} /\left(\mathrm{mol} \cdot \AA^{2}\right)$ per cycle. The fifth cycle consists of 10,000 steps of unrestrained minimization before the heating process. The cutoff distance used for the non-bonded interactions was $10 \AA$. The SHAKE algorithm [40] was used to restrain the bonds containing hydrogen atoms; (2) Each energy-minimized structure was heated over 120 ps from 0 to $300 \mathrm{~K}$ (with a temperature coupling of $0.2 \mathrm{ps}$ ), while the positions of Mad2 protein were restrained with a small value of $25 \mathrm{kcal} /\left(\mathrm{mol} \cdot \AA^{2}\right)$. The constant volume was maintained during the processes; (3) The unrestrained equilibration of $200 \mathrm{ps}$ with constant pressure and temperature conditions was carried out for each system. The temperature and pressure were allowed to fluctuate around $300 \mathrm{~K}$ and 1 bar, respectively, with the corresponding coupling of 0.2 ps. For each simulation, an integration step of 2 fs was used; and (4) Finally, conventional molecular dynamics (CMD) runs of 50 ns for the $\mathrm{C}-\mathrm{Mad} 2$ and the O-Mad2 models were carried out, respectively, by following the same protocol. 


\subsection{Targeted Molecular Dynamics Simulation}

Targeted MD simulation [41,42] is a method to observe large-scale conformational transitions between two known end-point conformations of a molecule. A restraint energy term was added to the energy function proportional to the square of the difference, which may be characterized as the mass-weighted root-mean-square deviation (RMSD) of the current structure to the target structure in terms of atomic positions. The functional form of the restraint energy can be written as:

$$
E_{\mathrm{TMD}}=\frac{1}{2} k N\left[R M S D(t)-R M S D_{0}(t)\right]^{2}
$$

where, $k$ is the harmonic force constant per atom, $N$ is the number of the restrained atoms, $R M S D(t)$ is the root-mean-square deviation of the simulated structure at time $t$ relative to the target structure, and $R M S D_{0}(t)$ is the prescribed target $R M S D$ value at time $t$ that decreases to zero linearly with time to drive the system from an initial structure to the target structure. To obtain the appropriate small force constant $k$, four independent, short (4 ns) TMD simulations were performed using different force constants of $0.2,0.5,0.8$ and $1.0 \mathrm{kcal} /\left(\mathrm{mol} \cdot \AA^{2}\right)$, respectively. As shown in Figure S3 for the conversion of O-Mad2 to C-Mad2, three out of four simulations reached a $R M S D$ value of $<2.0 \AA$ extracting from the backbone atoms of the Mad2 protein. Therefore, $k=0.5 \mathrm{kcal} /\left(\mathrm{mol} \cdot \AA^{2}\right)$ was chosen as the lowest harmonic force constant to apply onto all the backbone atoms of the Mad2 protein to bias the trajectories toward the target structure. All the other atoms in the Mad2 protein were assumed to move freely and to rearrange at their convenience to let the protein reach its equilibrium under this constraint. The weighted RMSDs along the TMD trajectories from four, 4 ns independent simulations showed that each process could follow the same conformational transition pathway, which indicated that the external forces and initial velocities did not affect the transition pathway. Therefore, the final TMD simulations were performed with the $10 \mathrm{~ns}$ transition TMD, followed by $20 \mathrm{~ns}$ of equilibration with an integration step of 2 ps. To test the mutual conversion property between the O-Mad 2 and $\mathrm{C}-\mathrm{Mad} 2$ states, the TMD simulations were performed in an $\mathrm{O}-\mathrm{Mad} 2 \leftrightarrow \mathrm{C}-\mathrm{Mad} 2$ bidirectional fashion.

\subsection{Correlation of Atomic Motions}

The dynamic feature of a protein and the extent of correlation of motions in the different regions of a protein were assessed via the calculation of cross-correlation coefficients, $\mathrm{C}(i, j)$, as follows:

$$
C(i, j)=\left(\Delta r_{i} \times \Delta r_{j} /\left[\left(\Delta r_{i}^{2}\right)\left(\Delta r_{j}^{2}\right)\right]^{1 / 2}\right.
$$

In the equation, $\Delta r_{i}$ and $\Delta r_{j}$ are the displacement vectors for $\mathrm{C} \alpha$ atoms of residues $i$ and $j$, respectively, and the angle brackets denote the ensemble averages [36,43]. In the present study, the correlation coefficients were averaged over the regions of protein, and the resulting cross-correlation coefficients are presented in the form of a two-dimensional graph. These structure analyses in the present work were calculated by using the PTRAJ module of the AMBER 9 program.

\subsection{Surface Analyses}

Shape descriptors representing protein structure, such as depth, surface curvature, extreme elevation, surface area, and volume, have been used extensively to identify, study, and compare 
protein-ligand interactions, protein-protein interactions, and the respective binding sites. We used the Computed Atlas of Surface Topography of proteins (CASTp) program (http://sts.bioengr.uic.edu/castp/) [44] to determine the binding pocket. The CASTp program uses weighted Delaunay triangulation and the alpha complex for shape measurements. It provides identification and measurements of surface accessible pockets as well as interior inaccessible cavities for proteins and other molecules. It analytically measures the area (in $\AA^{2}$ ) and volume (in $\AA^{3}$ ) of each pocket and cavity, both in solvent accessible surface (SA, Richards' surface) and molecular surface (MS, Connolly's surface) [44]. In our work, the probe radius was set to the default value (1.4 $\AA$ ). The detected pockets from using these algorithms are ranked with their volumes and areas. For ligand molecules, internal protein cavities appear to be a favored binding site, and the cavity volume may play an important role in the strength of the guest molecule-host cavity interaction. In CASTp analysis, we chose the Cdc20-binding pockets for our analysis.

\section{Conclusions}

Conventional molecular dynamics simulations and targeted molecular dynamics simulations were performed to address the stable structure characteristics for the open and closed Mad2 states and the dynamic transition mechanism of the two states, respectively. The results of this study show that the predominant structural differences between the stable open and closed Mad2 states were the transformations of the $\beta 7 / 8$ sheet and the $\beta 1$ strand with the rearrangement of $\beta 7-\beta 8-\beta 6-\beta 4-\beta 5-\beta 1$ to $\beta 6-\beta 4-\beta 5-\beta 8 "-\beta 8$ '. The results for the transition mechanism investigation demonstrated that the structural transition from the open Mad2 state to the closed one could occur via an intermediate. The structural change characteristics along the transition pathway indicate that the $\beta 1$ strand and the $\beta 7 / 8$ sheet unfold first, then move, and finally form an additional helix and a new $\beta 8^{\prime \prime} / 8^{\prime}$ sheet via the intermediate. Particularly, the $\beta 1$ strand traversing through the $\beta 5-\alpha \mathrm{C}$ loop is a prerequisite for the $\beta 7 / 8$ translocation. The conformation analysis for the intermediate indicates that the movement of the $\beta 6 / 4 / 5$ sheet close to the $\alpha \mathrm{C}$ helix causes the exposure of the $\beta 6$ strand and an increase in size of the active pocket, and favors the binding of the Mad2 protein to $\mathrm{Cdc} 20$. This observation reveals the critical function of interconversion of the two states in $\mathrm{APC} / \mathrm{C}$ inhibition. Correlation analysis reveals that the allosteric network occurs from the $\beta 1$ strand to the $\beta 7 / 8$ sheet via the $\beta 5-\alpha \mathrm{C}$ loop and the $\beta 6 / 4 / 5$ sheet in the conversion of the open $\operatorname{Mad} 2$ state to the closed Mad2 state. The present investigation provides useful insights into understanding the dynamics of the transition mechanism of the active closed Mad2 state to the open Mad2 state.

\section{Acknowledgments}

The authors acknowledge research support from the National Science Foundation of China (No. 20973024, 21073015, 21131003 and 21271029); and the Major State Basic Research Development Programs (grant No. 2011CB808500). 


\section{Author Contributions}

Conception and design: Y.W. and G.C.; Collection and assembly of data: C.L.; Data analysis and interpretation: C.L. and Y.Z.; and Manuscript writing: C.L. and Y.W.

\section{Conflicts of Interest}

The authors declare no conflict of interest.

\section{References}

1. Luo, X.; Yu, H. Protein metamorphosis: The two-state behavior of Mad2. Structure 2008, 16, 1616-1625.

2. Musacchio, A.; Salmon, E.D. The spindle-assembly checkpoint in space and time. Nat. Rev. Mol. Cell Biol. 2007, 8, 379-393.

3. Cleveland, D.W.; Mao, Y.; Sullivan, K.F. Centromeres and kinetochores: From epigenetics to mitotic checkpoint signaling. Cell 2003, 112, 407-422.

4. Skladanowski, A.; Bozko, P.; Sabisz, M. DNA structure and integrity checkpoints during the cell cycle and their role in drug targeting and sensitivity of tumor cells to anticancer treatment. Chem. Rev. 2009, 109, 2951-2973.

5. Du, Y.; Yin, F.; Liu, C.; Hu, S.; Wang, J.; Xie, H.; Hong, L.; Fan, D. Depression of MAD2 inhibits apoptosis of gastric cancer cells by upregulating Bcl-2 and interfering mitochondrion pathway. Biochem. Biophys. Res. Commun. 2006, 345, 1092-1098.

6. Musacchio, A.; Hardwick, K.G. The spindle checkpoint: Structural insights into dynamic signalling. Nat. Rev. Mol. Cell Biol. 2002, 3, 731-741.

7. Peters, J.M. The anaphase promoting complex/cyclosome: A machine designed to destroy. Nat. Rev. Mol. Cell Biol. 2006, 7, 644-656.

8. Ditchfield, C.; Johnson, V.L.; Tighe, A.; Ellston, R.; Haworth, C.; Johnson, T.; Mortlock, A.; Keen, N.; Taylor, S.S. Aurora B couples chromosome alignment with anaphase by targeting BubR1, Mad2, and Cenp-E to kinetochores. J. Cell Biol. 2003, 161, 267-280.

9. Shah, J.V.; Cleveland, D.W. Waiting for anaphase: Mad2 and the spindle assembly checkpoint. Cell 2000, 103, 997-1000.

10. Guardavaccaro, D.; Frescas, D.; Dorrello, N.V.; Peschiaroli, A.; Multani, A.S.; Cardozo, T.; Lasorella, A.; Iavarone, A.; Chang, S.; Hernando, E.; et al. Control of chromosome stability by the $\beta$-TrCP-REST-Mad2 axis. Nature 2008, 452, 365-369.

11. Nezi, L.; Rancati, G.; de Antoni, A.; Pasqualato, S.; Piatti, S.; Musacchio, A. Accumulation of Mad2-Cdc20 complex during spindle checkpoint activation requires binding of open and closed conformers of Mad2 in Saccharomyces cerevisiae. J. Cell Biol. 2006, 174, 39-51.

12. Kulukian, A.; Han, J.S.; Cleveland, D.W. Unattached kinetochores catalyze production of an anaphase inhibitor that requires a Mad2 template to prime Cdc20 for BubR1 binding. Dev. Cell 2009, 16, 105-117.

13. Wickliffe, K.; Williamson, A.; Jin, L.; Rape, M. The multiple layers of ubiquitin-dependent cell cycle control. Chem. Rev. 2009, 109, 1537-1548. 
14. Yang, M.; Li, B.; Liu, C.-J.; Tomchick, D.R.; Machius, M.; Rizo, J.; Yu, H.; Luo, X. Insights into $\operatorname{mad} 2$ regulation in the spindle checkpoint revealed by the crystal structure of the symmetric mad2 dimer. PLoS Biol. 2008, 6, e50.

15. Takahashi, T.; Haruki, N.; Nomoto, S.; Masuda, A.; Saji, S.; Osada, H. Identification of frequent impairment of the mitotic checkpoint and molecular analysis of the mitotic checkpoint genes, hsMAD2 and p55CDC, in human lung cancers. Oncogene 1999, 18, 4295-4300.

16. Bharadwaj, R.; Yu, H. The spindle checkpoint, aneuploidy, and cancer. Oncogene 2004, 23, 2016-2027.

17. Wang, X.; Jin, D.-Y.; Ng, R.W.; Feng, H.; Wong, Y.C.; Cheung, A.L.; Tsao, S.W. Significance of MAD2 expression to mitotic checkpoint control in ovarian cancer cells. Cancer Res. 2002, 62, 1662-1668.

18. Kops, G.J.; Weaver, B.A.; Cleveland, D.W. On the road to cancer: Aneuploidy and the mitotic checkpoint. Nat. Rev. Cancer 2005, 5, 773-785.

19. Murzin, A.G. Metamorphic proteins. Science 2008, 320, 1725-1726.

20. Luo, X.; Tang, Z.; Xia, G.; Wassmann, K.; Matsumoto, T.; Rizo, J.; Yu, H. The Mad2 spindle checkpoint protein has two distinct natively folded states. Nat. Struct. Mol. Biol. 2004, 11, 338-345.

21. Yang, M.; Li, B.; Tomchick, D.R.; Machius, M.; Rizo, J.; Yu, H.; Luo, X. p31comet blocks Mad2 activation through structural mimicry. Cell 2007, 131, 744-755.

22. Mapelli, M.; Massimiliano, L.; Santaguida, S.; Musacchio, A. The Mad2 conformational dimer: Structure and implications for the spindle assembly checkpoint. Cell 2007, 131, 730-743.

23. Luo, X.; Fang, G.; Coldiron, M.; Lin, Y.; Yu, H.; Kirschner, M.W.; Wagner, G. Structure of the Mad2 spindle assembly checkpoint protein and its interaction with Cdc20. Nat. Struct. Mol. Biol. 2000, 7, 224-229.

24. Luo, X.; Tang, Z.; Rizo, J.; Yu, H. The Mad2 spindle checkpoint protein undergoes similar major conformational changes upon binding to either Mad1 or Cdc20. Mol. Cell 2002, 9, 59-71.

25. Sironi, L.; Mapelli, M.; Knapp, S.; de Antoni, A.; Jeang, K.-T.; Musacchio, A. Crystal structure of the tetrameric Mad1-Mad2 core complex: Implications of a "safety belt" binding mechanism for the spindle checkpoint. EMBO J. 2002, 21, 2496-2506.

26. Mapelli, M.; Musacchio, A. MAD contortions: Conformational dimerization boosts spindle checkpoint signaling. Curr. Opin. Struct. Biol. 2007, 17, 716-725.

27. Fava, L.L.; Kaulich, M.; Nigg, E.A.; Santamaria, A. Probing the in vivo function of Mad1: C-Mad2 in the spindle assembly checkpoint. EMBO J. 2011, 30, 3322-3336.

28. Simonetta, M.; Manzoni, R.; Mosca, R.; Mapelli, M.; Massimiliano, L. The influence of catalysis on Mad2 activation dynamics. PLoS Biol. 2009, 7, e1000010.

29. Vink, M.; Simonetta, M.; Transidico, P.; Ferrari, K.; Mapelli, M.; de Antoni, A.; Massimiliano, L.; Ciliberto, A.; Faretta, M.; Salmon, E.D.; et al. In vitro FRAP identifies the minimal requirements for Mad2 kinetochore dynamics. Curr. Biol. 2006, 16, 755-766.

30. De Antoni, A.; Pearson, C.G.; Cimini, D.; Canman, J.C.; Sala, V.; Nezi, L.; Mapelli, M.; Sironi, L.; Faretta, M.; Salmon, E.D.; et al. The Mad1/Mad2 complex as a template for Mad2 activation in the spindle assembly checkpoint. Curr. Biol. 2005, 15, 214-225. 
31. Lad, L.; Lichtsteiner, S.; Hartman, J.J.; Wood, K.W.; Sakowicz, R. Kinetic analysis of Mad2-Cdc20 formation: Conformational changes in Mad2 are catalyzed by a C-Mad2-ligand complex. Biochemistry 2009, 48, 9503-9515.

32. Mapelli, M.; Filipp, F.V.; Rancati, G.; Massimiliano, L.; Nezi, L.; Stier, G.; Hagan, R.S.; Confalonieri, S.; Piatti, S.; Sattler, M. Determinants of conformational dimerization of Mad2 and its inhibition by p31comet. EMBO J. 2006, 25, 1273-1284.

33. Binkowski, T.A.; Naghibzadeh, S.; Liang, J. CASTp: Computed atlas of surface topography of proteins. Nucleic Acids Res. 2003, 31, 3352-3355.

34. Liang, J.; Woodward, C.; Edelsbrunner, H. Anatomy of protein pockets and cavities: Measurement of binding site geometry and implications for ligand design. Protein Sci. 1998, 7, 1884-1897.

35. Laskowski, R.A.; Luscombe, N.; Swindells, M.B.; Thornton, J.M. Protein clefts in molecular recognition and function. Protein Sci. 1996, 5, 2438-2452.

36. Case, D.; Darden, T.; Cheatham, T., III; Simmerling, C.; Wang, J.; Duke, R.; Luo, R.; Merz, K.; Pearlman, D.; Crowley, M. AMBER 9; University of California: San Francisco, CA, USA, 2006.

37. Lee, M.C.; Duan, Y. Distinguish protein decoys by using a scoring function based on a new AMBER force field, short molecular dynamics simulations, and the generalized born solvent model. Proteins 2004, 55, 620-634.

38. Duan, Y.; Wu, C.; Chowdhury, S.; Lee, M.C.; Xiong, G.; Zhang, W.; Yang, R.; Cieplak, P.; Luo, R.; Lee, T. A point-charge force field for molecular mechanics simulations of proteins based on condensed-phase quantum mechanical calculations. J. Comput. Chem. 2003, 24, 1999-2012.

39. Wang, J.; Wolf, R.M.; Caldwell, J.W.; Kollman, P.A.; Case, D.A. Development and testing of a general amber force field. J. Comput. Chem. 2004, 25, 1157-1174.

40. Miyamoto, S.; Kollman, P.A. SETTLE: An analytical version of the SHAKE and RATTLE algorithm for rigid water models. J. Comput. Chem. 1992, 13, 952-962.

41. Schlitter, J.; Engels, M.; Krüger, P.; Jacoby, E.; Wollmer, A. Targeted molecular dynamics simulation of conformational change-application to the $\mathrm{T} \leftrightarrow \mathrm{R}$ transition in insulin. Mol. Simul. 1993, 10, 291-308.

42. Schlitter, J.; Engels, M.; Krüger, P. Targeted molecular dynamics: A new approach for searching pathways of conformational transitions. J. Mol. Graph. 1994, 12, 84-89.

43. Sadiq, S.K.; de Fabritiis, G. Explicit solvent dynamics and energetics of HIV-1 protease flap opening and closing. Proteins 2010, 78, 2873-2885.

44. Dundas, J.; Ouyang, Z.; Tseng, J.; Binkowski, A.; Turpaz, Y.; Liang, J. CASTp: Computed atlas of surface topography of proteins with structural and topographical mapping of functionally annotated residues. Nucleic Acids Res. 2006, 34, W116-W118.

(C) 2014 by the authors; licensee MDPI, Basel, Switzerland. This article is an open access article distributed under the terms and conditions of the Creative Commons Attribution license (http://creativecommons.org/licenses/by/3.0/). 